\title{
La struttura di personalità delle figure sanitarie nel lavoro psicologico-psichiatrico
}

Personality structure in healthcare professionals in psychology-psychiatry

CMI 2020; 14(1): 21-26

http://dx.doi.org/10.7175/cmi.v14i1.1477

\section{NOTE INTRODUTTIVE}

Il complesso lavoro psicologico-psichiatrico ha bisogno che gli operatori sanitari dispongano di personalità strutturate e ragionevolmente mature. Questa caratteristica risulta essenziale perché tale operatività possa raggiungere i risultati migliori per il gruppo sanitario e quindi per il paziente e costituisce, pertanto, un'importante dimensione finale dell'attività terapeutica. Attraverso questa modalità è possibile capire i bisogni del paziente costruendo un legame capace di uno sviluppo positivo. La comprensione del significato delle esperienze che hanno agevolato lo sviluppo della psicopatologia diventa così un passaggio significativo, non eliminabile o restringibile. Di fatto, una relazione terapeutica non basata o che lasci in ombra questa prassi è deputata a un probabile fallimento.

Non casualmente, questa tipologia di lavoro si basa essenzialmente sulla capacità degli operatori sanitari di raggiungere il percorso di vita del paziente e non il tragitto inverso. In caso contrario, questa persona avvertirebbe di essere ancora un "nomade dell'esistenza", percezione che bloccherebbe o ostacolerebbe ogni minimo tentativo di cambiamento.

Per queste ragioni gli operatori psicologico-psichiatrici devono disporre di strutture di personalità fornite di un'esperienza capace di comprendere il vissuto del paziente, evitando una relazione formale che sarebbe nociva e inconcludente.

\section{LA STRUTTURA DELL'OPERATORE SANITARIO NEL CAMPO PSICOLOGICO-PSICHIATRICO}

In questo campo, l'operatore sanitario deve quindi possedere una struttura basata su presupposti di personalità che comprendano una serie di caratteristiche basate su una sequenza interna non alterabile. Tale successione si evidenzia nel sentire $>$ pensare $>$ comunicare $>$ comportarsi. Questa sequenza permette alla capacità personale di poter affrontare il mondo circostante e i suoi problemi con un ragionevole buonsenso e padronanza.

È importante, inoltre, sottolineare che delle quattro fasi il "sentire" rappresenta l'elemento essenziale che caratterizza profondamente quelle successive. Infatti, il "sentire" è in grado di condizionare fortemente le altre tre fasi, ponendole nella condizione di rispondere quasi automaticamente al suo contenuto (povero o ricco che sia di esperienze, di valori, di principi etici, ecc.). In questa prospettiva, il "sentire" assume una sua sovranità psicodinamica regolativa di tutte le altre manifestazioni interne (pensiero) ed esterne (comunicazione e comportamento).

Tale susseguirsi prende forma precocemente (infanzia), consolidandosi attraverso le esperienze degli stadi evolutivi successivi (adolescenza e maturità) e al significato che viene attribuito alle stesse. Il "Magaz-

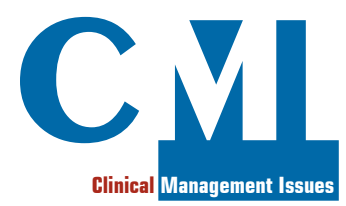

Editoriale

Psicoterapeuta, La Spezia

Corresponding author Roberto Infrasca roberto.infrasca@libero.it

Received: 15 April 2020 Accepted: 24 April 2020 Published: 15 July 2020 
zino degli Elementi Esperienziali” (MEE) contiene questi tasselli esperienziali (umani, relazionali, lavorativi, culturali, sessuali, ecc.) dai quali l'individuo attinge fattori e panorami esistenziali che gli permettono di comporre nuove e oggettive elaborazioni e vissuti della realtà.

Un'esperienza, per essere archiviata definitivamente nel MEE, abbisogna di alcune essenziali caratteristiche. La prima viene rintracciata nel vissuto implicato nell'esperienza stessa, vale a dire che rappresenti per la persona una notevole importanza soggettiva, condizione per cui diviene parte integrante della memoria storica individuale. L'altra si evidenzia nell'accettazione dell'esperienza negativa con un sentire imparziale che, nel rispetto della propria dignità, l'individuo ritiene che comunque arricchirà la sua memoria storica.

Partendo dal presupposto che il MEE si organizza nell'infanzia, questo stadio evolutivo diviene essenziale a formare un'architettura mentale "fluida" o "rigida", una visione del mondo e degli altri fondata particolarmente su elementi realistici oppure illusori e fantastici, una capacità relazionale prevalentemente organizzata su presupposti esperienziali soggettivi e obiettivi oppure soltanto sui propri bisogni e desideri individualistici.

Peraltro, in assenza di una memoria storica, qualsiasi esperienza già vissuta risulterebbe nell'attualità del tutto nuova, situazione che metterebbe l'individuo nella condizione di non sapere come valutarla, gestirla e comportarsi. Qualora tale esperienza non venga interiorizzata nel MEE (la memoria storica), ogni ulteriore e analoga situazione continuerà a essere vissuta come nuova $\mathrm{e}$ indecifrabile.

Quanto descritto significa che il MEE risulta di alta significatività vitale per l'individuo, un attendibile "suggeritore" interno capace di orientare il modello mentale, comunicativo e comportamentale verso situazioni che prevedano la riduzione (a volte l'annullamento) di errori e quindi del probabile fallimento soggettivo. Il MEE assume così un profilo di notevole importanza nell'esistenza individuale (quindi relazionale), essendo in grado di permettere a una persona di accumulare esperienza.

Senza esperienza l'essere umano "nascerebbe" ogni giorno. Senza esperienza non sarebbe in grado di decidere. Senza esperienza si perderebbe nelle circostanze e situazioni quotidiane che la vita propone, che risulte- rebbero non distintamente comprensibili e quindi valutabili.

Se nel MEE risiedono poche e scarne esperienze e ricordi persistenti delle stesse, queste non possono che presentare lo stesso profilo, situazione che pone il Magazzino degli Elementi Esperienziali nella situazione di impoverirsi e così l'individuo che lo possiede. Le esperienze e la memoria, peraltro, permettono la "plasticità del cervello", vale a dire la capacità del cambiamento delle relazioni tra neuroni e quindi quello personologico e comportamentale.

Tali affermazioni sono ampiamente verificate nelle ricerche di Kandel [1,2]. Premio Nobel per la Medicina (2000), l'autore sostiene che l'essere umano sia il prodotto dell'interazione tra le sue esperienze, i suoi ricordi el'apprendimento conseguente. Kandel scoprì che il nostro apprendimento non avviene attraverso la modificazione dei neuroni, ma rafforzando le sinapsi (connessioni tra i neuroni) o costruendone di nuove, dinamica che modifica continuamente la nostra mente (cervello) rispetto alle esperienze vissute (processo tipico per la memoria a lungo termine - MLT, che riflette la complessità del MEE).

Una breve parentesi può essere utile per comprendere meglio l'argomento trattato. La comunicazione si divide sostanzialmente in verbale e non verbale, ma pare importante inserire anche unulteriore e analoga suddivisione tra detto e non detto. I1 "detto" rappresenta la parte della realtà sentita e comunicata. Il "non detto" è la realtà rimanente che invece non viene comunicata e che ostacola profondamente la comprensione da parte degli altri, della persona che usa questa tipologia di linguaggio [3].

La prima modalità comunicativa chiarisce e diviene di prossimità, la seconda nasconde e allontana. Lesperienza professionale permette di percentualizzare tali modalità nel modo seguente: "detto" tra il $20 \%$ e il $15 \%$, "non detto" tra 1'80\% e 1'85\%, mentre una comunicazione ragionevolmente umana e genuina prevedrebbe percentuali inverse. I1 "non detto" rende quindi la persona che lo usa anonima e apolide a livello comprensivo ed esistenziale, occultando la vera sostanza personale e quindi una lettura ragionevole e accessibile della stessa. Tale voluta indifferenziazione personologica necessita così di ulteriori e impegnative rielaborazioni tese a completare il profilo umano comunicato, rendendo impossibile la lettura personologica nell' $80-85 \%$ delle situazioni. 
Peraltro, il pensiero deve essere necessariamente comunicato: in caso contrario diviene un "pensiero afono", che non assume una funzione e una coloritura personale, relazionale e culturale, quindi inutile a livello umano ed esistenziale.

Il MEE, quindi, risulta il contenitore di tutti gli engrammi personali accumulati, che divengono la memoria storica dell'individuo senza il quale lo stesso "non è". La scarsità di engrammi comporta un pensiero elementare, mentre la numerosità e la complessità degli engrammi implicano un pensiero complesso, che spazia per orizzonti complicati e ampi.

Senza memoria storica una persona (o una comunità) rischia di perdere e smarrire il significato profondo della propria identità culturale e civile. Tra le diverse tipologia di memoria, solo quella critica è in grado di selezionare e discernere $\mathrm{i}$ ricordi in base a un atteggiamento rivolto a capire la vicenda umana (soggettiva e collettiva) per come si è sviluppata lungo la linea del tempo.

Nelle caratteristiche assunte dal MEE, risiedono le basi del pensiero pensante o solo di forme alternative di pensiero. In questo terreno, il metodo educativo familiare diviene il modellatore del pensiero infantile (poi adolescenziale e maturo). Le sistematiche sollecitazioni genitoriali orientate a dare al bambino una molteplicità di panorami esistenziali è all'origine della possibilità di una "multi-esperienza" oppure di una "monoesperienza" della realtà personale e interpersonale di questa piccola e ancora informe dimensione umana. Di fatto, mentre il pensiero infantile risulta molto duttile e quindi plasmabile, quello adolescenziale ha un profilo meno flessibile e quello maturo si presenta, generalmente, come maggiormente stabile (qualsiasi sia la sua valenza o orientamento).

L'empatia umana deriva principalmente dalle esperienze immagazzinate nel MEE, divenendo un diretto prodotto di questo. Dalla premessa, si comprende come la struttura caratterologica dell'operatore sanitario nel lavoro psicologico-psichiatrico debba necessariamente essere il più possibile conforme alle prerogative delineate.

\section{IL RUOLO DELL'EMPATIA NEL LAVORO PSICOLOGICO- PSICHIATRICO}

L'empatia, intesa come consapevolezza dei pensieri e dei sentimenti di un'altra persona, del "sentire" il mondo come questo lo vede e lo vive condividendone aspetti emozionali e cognitivi, diventa così indispensabile. L'empatia, non casualmente, è un requisito fondamentale nella psicoterapia, pratica nella quale il terapeuta partecipa profondamente all'esperienza del paziente pur conservando un'indipendenza emotiva dallo stesso. Lorigine dell'empatia viene così rintracciata nella conformazione del MEE, nella sua capacità di estendersi verso un panorama umano vasto e multiforme.

Il lavoro psicologico-psichiatrico non può quindi essere svolto positivamente senza questa essenziale capacità di base dell'operatore sanitario in quanto, deficitaria di questa caratteristica, la relazione terapeutica sarebbe solamente personale e non interpersonale. La preparazione clinica dell'operatore è decisamente necessaria ma non bastevole per un effettivo lavoro in questa delicata attività. Tutte le attività che prevedono un'attività terapeutica sull'uomo, così, non possono prescindere da alcuni essenziali criteri di contributo.

Tra questi, possono essere elencati:

- la capacità di mettere in ombra le proprie aspettative e i propri bisogni soggettivi;

- l'attitudine a lavorare, se non unicamente, ma in maniera prevalente sui bisogni dell'Altro;

- la tendenza a vedere nel paziente lo scopo principale e non un mezzo per il proprio successo;

- il bisogno di avvalersi delle diverse professionalità insite nel gruppo per aumentare la propria competenza e la coesione di questo nell'intervento sul disagio psichico. Visto in quest'ottica il lavoro psicologicopsichiatrico (come in altri) assume così un valenza importante pur tra i rilevanti ostacoli che tale attività prevede. Comprendere il tragitto vitale che ha percorso il paziente per diventare tale non è facile né veloce. Di fatto, richiede un'interazione e un'introspezione lunga e quasi sempre dolorosa, che prevede l'importante domanda: chi sono io e chi è lui? Posta in questo scenario, tale attività assume un profilo gratificante e difficoltoso, ed è qui che entra in campo la personalità dell'operatore sanitario. Se pur professionalmente possa essere anche soddisfacente, affrontare la struttura psicopatologica risulta difficoltoso. Questa è una consapevolezza individuale attraverso la quale l'operatore sanitario proseguirà il suo tragitto professionale, mentre in caso contrario sarà indotto a un discreto ma inesorabile abbandono per non mettere 
in forse le proprie "sicurezze" e, con queste, la propria struttura di personalità.

Tali affermazioni, infatti, trovano condivisione in molti lavori nella letteratura di ricerca che, pur partendo da ambiti e approcci differenziati, approdano a identiche conclusioni sull'importanza della personalità e della tipologia comunicativa messa in atto dall'operatore all'interno del lavoro psicologico-psichiatrico [4-7].

Il lavoro psicologico-psichiatrico ha quindi bisogno di operatori sanitari che dispongano di una struttura di personalità "ragionevolmente organizzata", per quanto umanamente possibile. Nella realtà, invece, è possibile trovare architetture caratteriali negative e positive. Di seguito proponiamo una panoramica delle stesse.

\section{ASPETTI NEGATIVI DELLA PERSONALITÀ DELL'OPERATORE CHE SUOLGE IL LAVORO SANITARIO}

Tra le caratteristiche ritenute negative viene rintracciata l'empatia scambiata per confidenzialità, per collusione con il paziente, l'attingere (inconsapevolmente) dal paziente la propria autostima, la proiezione sul paziente dei propri problemi per curarli all'“esterno". In questo panorama, alcune caratteristiche psico-comportamentali dell'operatore assumono un ruolo determinante.

Principalmente troviamo le note narcisistico-onnipotenti presenti nelle situazioni che prevedono una relazione con la psicopatologia. La presenza di tali tratti può condurre l'operatore a negare aspetti della realtà e quindi del paziente stesso, a causa del mancato riconoscimento o della non accettazione dei propri limiti (possibilità di dare risposte a tutto), originando nel paziente un'illusoria aspettativa di soluzione dei suoi problemi che determina una sua passività e la frequente cronicizzazione della relazione tra operatore e paziente.

L'anticipazione dei bisogni è un meccanismo attraverso il quale l'operatore sposta sul paziente il proprio desiderio di sentirsi utile, con il rischio di bloccare la comunicazione dei suoi bisogni inespressi, frequentemente non individuati dalla figura sanitaria. La scarsa capacità di quest'ultima a tollerare i tempi comunicativi del paziente (che suscitano timori di inutilità), possono indurre l'operatore a un intervento soggettivo che invade il “territorio emotivo" dell'altro, restringendone il già ridotto spazio personale. In questa pro- spettiva, essere realmente un valido aiuto per l'altro significa imparare ad attendere rispettando i suoi tempi e i suoi silenzi.

La proiezione delle parti "buone e cattive" dell'operatore tale per cui la vicinanza o la distanza dal paziente realizza un rapporto non reale con il paziente ma con i fantasmi dei propri vissuti emotivi. Proprio la caratteristica professionale delloperatore (relazione tra due soggettività) che prevede la messa in atto della proiezione si verifica spesso, poiché la soggettività dell'operatore deve confrontarsi con tante altre soggettività (i pazienti) e questo stimola nel Sé vissuti personali e risposte psico-comportamentali (vicinanza o distanza) a seconda che i segmenti rievocati siano correlati a situazioni personali positive o negative, piacevoli o sgradite, appaganti o frustranti. Spostando sul paziente le proprie problematiche e tentando di risolverle attraverso lui, l'operatore avrà l'illusione di non viverle come appartenenti a se stesso.

L'eccedenza di autocentrismo (su di sé) o di eterocentrismo (sul paziente) implica un'assenza della realtà vissuta nella relazione terapeutica oppure vissuta interamente sul paziente e quindi fuori da sé, con l'inevitabile conseguenza di un'impossibilità dell'“ascolto". In realtà, nel primo caso l'operatore non può costruire una relazione di ascolto con il paziente poiché sottomesso ai bisogni del proprio sé, mentre nel secondo la negazione dei propri bisogni per un univoco ascolto di quelli del paziente può fare smarrire i confini del sé e della realtà professionale, presentando un'immagine di precarietà e di inadeguatezza che genera insicurezza, compromettendo la serenità della relazione.

\section{ASPETTI POSITIVI DELLA PERSONALITÀ DELL'OPERATORE CHE SVOLGE IL LAVORO SANITARIO}

Gli aspetti positivi della personalità dell'operatore che svolge il lavoro sanitario sono quindi basati essenzialmente su alcune dimensioni tipiche dell'Io adulto. Tra queste, si evidenzia la fiducia (fiducia in sé e negli altri), dimensione costruita soprattutto nelle prime fasi dell'età evolutiva, nei rapporti con gli adulti nei primi anni di vita e nell'importanza delle esperienze successive.

Sulla fiducia si basa la sicurezza, vale a dire la fiducia nelle proprie capacità di affrontare la realtà e le difficoltà che la stessa implica senza sentirsi intimoriti. La sicurezza può essere definita nella sua forma più evoluta 
e consapevole come "autostima" (giudizio e valutazione di sé e delle proprie capacità).

L'autonomia è legata alla sicurezza e alla fiducia, raffigurando concretamente il vissuto di un Io separato dagli altri (originariamente dalle figure genitoriali), quindi in grado di affrontare la realtà, di assumersi responsabilità, di competere per la realizzazione di sé.

L'immagine di sé è un altro importante tratto caratterologico (anche per l'operatore sanitario). Il tragitto evolutivo della costruzione dell'Io implica una rappresentazione sempre più organizzata e molteplice di sé come essere distinto dagli altri in quanto dotato di una propria individualità. L'immagine di sé si configura come concetto di sé, vale a dire quale rappresentazione consapevole della propria individualità (sentimenti, valori, capacità, aspirazioni, affetti, ecc.).

Ai tratti di personalità citati va aggiunto il principio di realtà inteso come «una funzione preconscia costantemente attiva che automaticamente sceglie le nostre esperienze per orientarci nel comprendere se si tratta di una percezione esterna o intrapsichica - una vera percezione dei sensi o un'illusione; un ricordo o un prodotto dell'immaginazione; $\mathrm{e}$ quale posizione occupi all'interno di queste categorie - un vero oggetto o il disegno di un oggetto, il ricordo di un fatto o il ricordo di una fantasia» [8].

In definitiva, una persona, per capire chi ha "di fronte a sé" deve capire chi ha "dentro di sé".

\section{CONCLUSIONI}

Il lavoro ha messo in luce come il Magazzino degli Elementi Esperienziali (MEE) coniughi il divenire di una persona e divenga importante nel lavoro psicologico-psichiatrico in quanto organizzatore interno che assume il ruolo di coordinatore della prassi messa in atto nella realtà dall'operatore sanitario.

Questa condizione strutturale della caratterologia soggettiva permette, oltre alle necessarie conoscenze cliniche, di muoversi fluidamente in una relazione terapeutica per niente semplice il cui esito non è scontato. Il "sentire" diviene così il contenitore deposita- rio di una serie di contenuti estremamente importanti (memoria storica delle esperienze, sentimenti, vissuti, valori, ecc.), "sapere" dal quale dipendono in modo strettamente dipendente il pensiero, la comunicazione e il comportamento.

Per tale ragione, un contenitore ricco e multiforme dà luogo a un pensiero altrettanto ricco e multiforme, a una comunicazione e un comportamento elaborati e sotto la sorveglianza del "sentire". Al contrario, un contenitore con contenuti mediocri o poveri determina un pensiero angusto e una comunicazione e un comportamento stereotipati e individualistici.

La prima condizione di base, peraltro, permetterebbe all'operatore di comprendere, attenuare e governare quelli che vengono definiti i rischi delle professioni sanitarie (per esempio le note narcisistico-onnipotenti, l'anticipazione dei bisogni, la proiezione e, in parte, il fenomeno del burn-out), mentre un MEE deficitario di una molteplicità esperienziale renderebbe la relazionalità rigida, formale e convenzionale, quindi antiterapeutica.

In questa prospettiva, l'esperienza personale storicizzata diviene essenziale poiché orienta in modo significativo quella professionale. Tale processo amplifica molto la risonanza delle informazioni cliniche formative, che divengono un importante supporto all'interno della relazione terapeutica messa in atto.

La presenza nell'operatore di quanto delineato rende lo stesso maggiormente consapevole di sé e del paziente (l'altro da sé), aumentando la probabilità che il lavoro terapeutico trovi un approdo soddisfacente per entrambi. Di fatto, la consapevolezza della propria personalità agevola parallelamente la consapevolezza della personalità del paziente (storia, vicissitudini, significato dell'espressività sintomatologica), divenendo un intervento terapeutico che si sviluppa "in verticale" e non solamente "in orizzontale".

Quanto argomentato diviene un attendibile indirizzo per i gruppi che svolgono un lavoro psicologico-psichiatrico, fornendo loro le determinanti di base che possono agevolare od ostacolare questa importante e delicata prassi terapeutica.

\section{Fonti di finanziamento}

Questo articolo è stato pubblicato senza il supporto di sponsor.

\section{Conflitti di interesse}

L'autore dichiara di non avere conflitti di interesse riguardanti gli argomenti trattati nel presente articolo. 


\section{BIBLIOGRAFIA}

1. Kandel ER. A new intellectual framework for psychiatry. Am J Psychiatry 1998; 155: 457-69; https://doi.org/10.1176/ajp.155.4.457

2. Kandel ER. Biology and the future of psychoanalysis: a new intellectual framework for psychiatry revisited. Am J Psychiatry 1999; 156: 505-24; https://doi.org/10.1176/ajp.156.4.505

3. Mehrabian A. Nonverbal communication. Chicago (USA, IL): Aldine-Atherton, 1972

4. Vigorelli M (a cura di). Il lavoro della cura nelle Istituzioni. Progetti, gruppi e contesti nell'intervento psicologico. Milano (Italy): Franco Angeli, 2005

5. Pellegrini P. Riflessioni sull'identità degli operatori in psichiatrica. Psicoterapia e scienze umane 2009; 4: 545-58

6. Lorettu L, Nivoli GC, Milia P, et al. Il colloquio in situazioni psichiatriche di crisi [Clinical interview in psychiatric difficult situations]. Riv Psichiatr 2017; 52: 150-7; https://doi. org/10.1708/2737.27907

7. Montinari G. Proteggere e comunicare in terapia psichiatrica. Disponibile all'indirizzo http:// www.giandomenicomontinari.it/index.php/pubblicazioni/proteggere-e-comunicare-in-terapiapsichiatrica/ (ultimo accesso luglio 2020)

8. Rapaport D. Le tecniche proiettive e la teoria del pensiero. In: Rapaport D.Il modello concettuale della psicoanalisi. Milano: Feltrinelli, 1977 